\author{
Christos C. Chamis
}

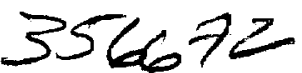

\title{
Probabilistic Composite Design
}

REFERENCE: Chamis, C. C., "Probabilistic Composite Design," Composite Materials: Testing and Design. Thirteenth Volume, ASTM STP 1242, S. J. Hooper, Ed., American Society for Testing and Materials, 1997, pp. 23-42.

\begin{abstract}
Probabilistic composite design is described in terms of a computational simulation. This simulation tracks probabilistically the composite design evolution from constituent materials, fabrication process, through composite mechanics and structural components. Comparisons with experimental data are provided to illustrate selection of probabilislic design allowables, test methods/specimen guidelines, and identification of in situ versus pristine strength. For example, results show that: in situ fiber tensile strength is $90 \%$ of its pristine strength; flat-wise long-tapered specimens are most suitable for setting ply tensile strength allowables: a composite radome can be designed with a reliability of 0.999999; and laminate fatigue exhibits wide-spread scatter at $90 \%$ cyclic-stress to static-strength ratios.
\end{abstract}

KEYWORDS: design methods, computer codes, design allowables, graphite fibers, test data, comparisons, in situ strength, testing guidelines, shear buckling, component reliability, laminate fatigue

Probabilistic composite design is simply the process required to evaluate the reliability of a specific design. The reliability of any specific design is determined by evaluating the probability that all structural design criteria are satisfied at pre-specified levels of probability. Evaluation of the probabilities for the various structural responses to satisfy the prespecified design criteria requires quantification of uncertainty ranges for each response. To quantify those uncertainty ranges in composite structural design, we need to have formal methods that trace the uncertainty ranges of all participating variables in the structural design. We-therefore must start with the constituent materials and continue introducing uncertainties expected to be present as we progress through the higher scales (micro, macro, laminate, and structure). Another important aspect in probabilistic composite structural design is verification. Substantial work has been reported for the predictive part of probabilistic composite structural design [1,2]. The objective of the present paper is to describe a multiscale probabilistic composite design method and comparison with measured data.

A multiscalc probabilistic composite design to be practical must be in the form of computational simulation. In order to be credible it must have appropriate verification at all scale levels. The procedure must be suitably illustrated to be instructive. The design must be representative of typical designs to add confidence in the method and simulation scheme used. In this article we introduce probabilistic structural analysis/design with a simple component in order to identify the essential elements. We continue with the description of the formal methods and their respective computer codes. We follow these up with applications for setting and/or deciding strength allowables for ply, laminate, and laminate with holes. We describe select extensions for fabrication implications, testing guidelines, and laminate fatigue. We conclude with a sample probabilistic composite design. Select refer-

'Senior aerospace scientist, NASA Lewis Research Center, Mail Stop 5-11, 21000 Brookpark Road, Cleveland, $\mathrm{OH} 44135-3191$. 
ences are cited for complimentary information, particularly for the equations used for composite mechanics, probabilistic structural analysis, and probabilistic sensitivities.

\section{Fundamental Concept}

It is instructive to describe some fundamental concepts of probabilistic structural analysis/ design by using a simple example. The simple example for that purpose is the evaluation of the tip displacement of a cantilever beam loaded at the free end as shown schematically at the top left of Fig. 1. The equation for predicting the tip displacement is shown under the schematic. This equation includes the fundamental parameters (primitive variables) that govern the tip displacement. For example, $P$ is the load, $l$ is the length, $E$ is the material stiffness, $b$ is the width, and $t$ is the thickness. These primitive variables can also be grouped in three generic categories: load $(P)$, geometry $(l, b$, and $t)$, and material $(E)$. Experience has shown that if we make several cantilever specimens there will be a scatter of values for each of those variables. The task, therefore, of probabilistic simulation is to account for the effects of that scatter on the displacement of the beam.

The task is considerably simplified when we recognize that (1) the tip displacement equation is the analogue of a physical testing machine, and (2) the scatter in the primitive variables, $P, l, b, t$, and $E$, can be assumed to be represented by simple and well known statistical distributions (Fig. 1, lower left). These distributions help us in two ways, as will become evident subsequently. In order to evaluate the effects of the uncertainties of the primitive variables on the tip displacement, we proceed as follows: Step 1: we decide on the range of the scatter in each primitive variable. This range in practical cases is established from experience, but for our simple example we assume that scatter for the modulus is between 172 and $214 \mathrm{GPa}(25$ and $31 \mathrm{mpsi}$ ); for the length, between 48.3 and $53.4 \mathrm{~cm}$ (19 and $21 \mathrm{in}$.); for the width, between 3.05 and $3.30 \mathrm{~cm}(1.20$ and $1.30 \mathrm{in}$.$) ; for the thickness,$ 2.41 and $2.67 \mathrm{~cm}(0.95$ and $1.05 \mathrm{in}$.); and for the load between 356 and $534 \mathrm{~N}$ ( 80 and $120 \mathrm{lb}$ ). It is important to note that the only test data we had were the mean values for the primitive variables. We assumed the range of the scatter. Note that the mean value for each primitive variable is where the vertical line drawn from the peak of the respective distribution intersects the horizontal line. Step 2: for each primitive variable in the equation we select randomly a value from within its respective scatter. Having the simple statistical distributions allows us to make the non-biased random selection. For example, the values selected randomly can be: $176 \mathrm{GPa}(25.5 \mathrm{mpsi})$ for $E, 52.8 \mathrm{~cm}(20.8 \mathrm{in}$.) for $1,3.23 \mathrm{~cm}$ (1.27 in.) for $b, 2.51 \mathrm{~cm}(0.99 \mathrm{in}$.) for $t$, and $512 \mathrm{~N}(115 \mathrm{lb})$ for $P$. Step 3: When we substitute these values in the equation, we get $0.334 \mathrm{~cm}(0.132 \mathrm{in}$.) for the tip deflection. Step 4: Repeat Step 3 for different sets of primitive variable values until sufficient data have been accumulated to plot the probability distribution graph (Fig. 1, upper right). For example, the mean value will be close to $0.305 \mathrm{~cm}(0.120$ in.). There is about $60 \%$ probability from the cumulative probabilistic graph to obtain the value of $0.334 \mathrm{~cm}(0.132 \mathrm{in}$.) calculated in Step 3.

When the data are generated in Step 4, as just described, it is called Direct Monte Carlo Simulation and generally requires a large number of simulations. Methods/algorithms have been developed to generate the two probability graphs for the displacement with a relative few number of simulations. One such method is known as the Fast Probability Integration (FPI) [3]. That method was used to generate the probability graphs (Fig. 1). Application of FPI requires input of mean value, scatter range, and probability density function of the scatter for each participating primitive variable. As already mentioned, the probabilistic simulation can be performed with known mean values and judiciously assumed scatter ranges for the primitive variables. 


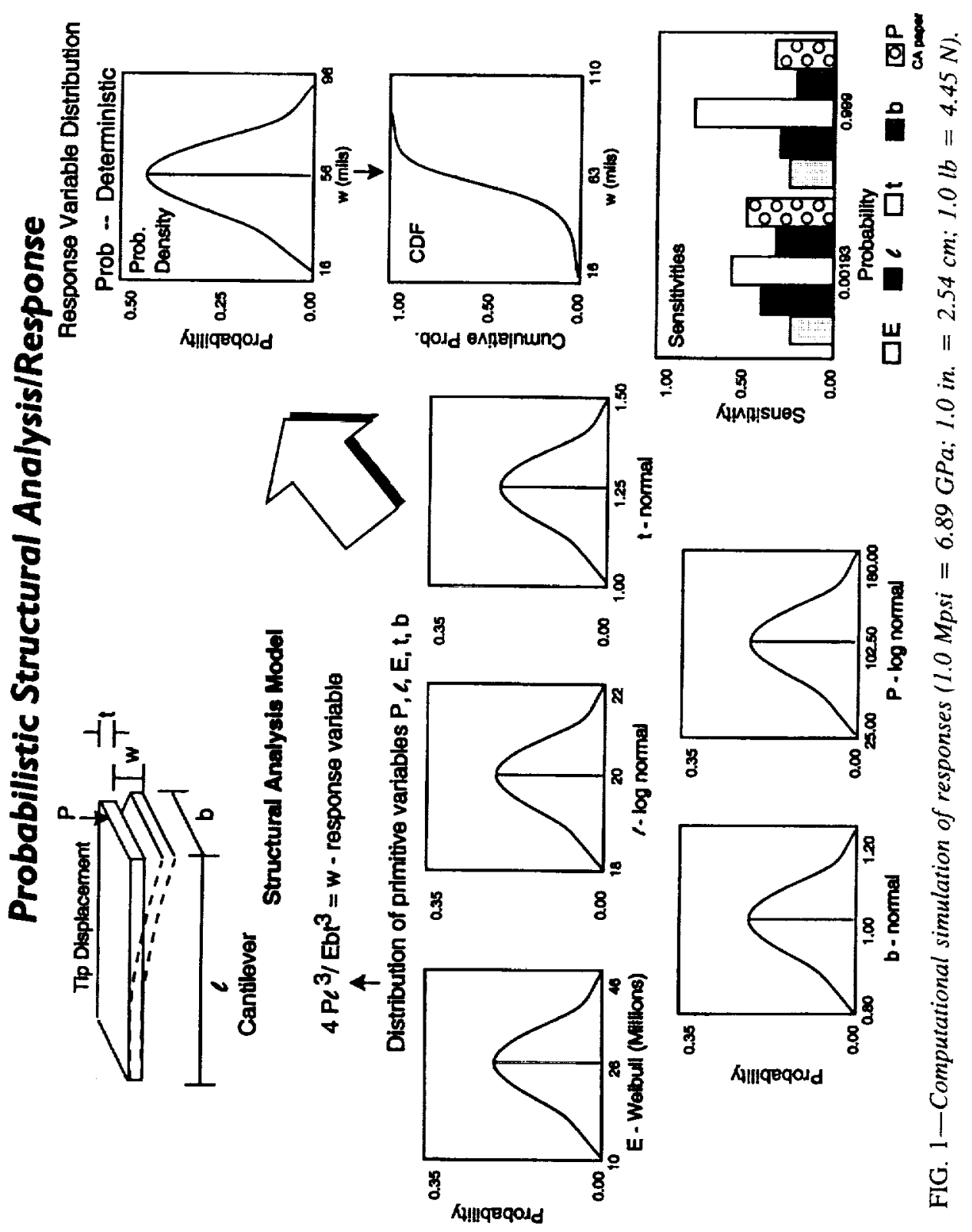


A byproduct of the FPI is the sensitivity factors (Fig. 1, lower right). These factors quantify and order the sensitivity of the cumulative dissolution function of the response variable to the uncertainty (scatter range) in the primitive variables. For our simple example. the load (primitive variable) has about the same effect on the tip displacement (response variable) as the geometry parameters (primitive variables) for low probability (less than $I$ in 1000), while the thickness (primitive variable) dominates at high probabilities (greater than 999 in 1000). More about sensitivities in later sections.

For composite structural components/structures, the above procedure is generalized as follows:

Step 1. Develop a finite element model of the entire structure with its boundary and load conditions.

Step 2. Use integrated composite mechanics to predict the composite properties starting with micromechanics and accounting for environmental effects.

Step 3. Identify the primitive variables. These will include constituent materials, fabrication process variables, structural parameters, loads, (including environment), boundary conditions, elc.

Step 4. Obtain/assume mean values, scatter range, and probabilistic distribution for each primitive variable.

Step 5. Randomly select values for each primitive variahle from their respective distributions.

Step 6. Conduct a finite clement analysis with the values selected in Step 5.

Step 7. Repeat Steps 5 and 6 several times for FPI use.

Step 8 . Use FPI to generate the probability distribution functions for the desired response, displacement, stress, frequency, etc.

The above generalized procedure is practical through computer codes as will be described subsequently.

\section{Source of Uncertainties in Composites}

We consider the schematic shown in Fig. 2 in order to identify sources of uncertainties in composites and therefore primitive variables. The schematic in Fig. 2 is a simplistic representation of composite fabrication process but contains most of the important variables for our purposes. All the items listed under the schematic constitute one or more primitive variables. For example, at any one point through the laminate thicknesses and for each ply in the laminate, there are as many primitive variables as there are constituent material properties but only single primitive variables for the other factors under the schematic. We will discuss these primitive variables further in the verification and application sections. Identify primitive variables at the lowest fundamental level and all other levels and then let the mechanics propagate their respective scatter to the desired response accounts automatically for correlations among variables that may be present at higher scales.

\section{Computational Simulation Method}

It is important to note that computational simulation methods are not unique. They very much depend on the experience of the researchers who develop these methods. The compulational simulation method used to obtain the results to be presented later is shown schematically (Fig. 3). This computational method is called (IPACS) for Integrated Probabilistic Assessment of Composite Structures. 


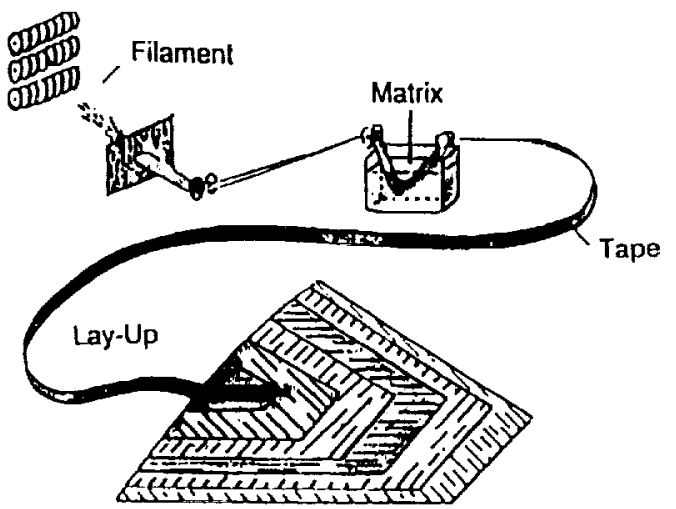

Fabrication Schernalic
- Constituents
- Fiber Misalignment
- Fiber Volume Ratio
- Void Volume Ratio
- Ply Orientation Angle
- Ply Thickness

FIG. 2--Sources of scaller-fabrication process.

IPACS was developed by merging two independent stand alone computer codes: (1) probabilistic composite mechanics and (2) probabilistic structural analysis. Probabilistic composite mechanics is simply the ICAN (Integrated Composite Analyzer) $\lceil 4\rceil$ computer code with provisions for uncertainties and FPI to generate the probabilistic distributions for composite or laminate properties at any scale. The details are described in Refs 5 and 6 . The reason to use proven deterministic codes is that the mean should be accurately predicted compared to measured data. Probahilistic structural analysis is simply (1) a deterministic general purpose finite element analysis upgraded to account for uncertainties in the structural primitive variables and (2) FPI to generate the probability distribution functions of the desired structural response with a relatively small number of simulations. The details of the prohabilistic finite element analysis and probahilistic sensitivities are described in Ref 7. Suffice to saly that the deterministic finite element analysis [7] accurately predicts all the usual and not so usual structural responses. It is very important to note that by using proven (credible) deterministic methods and codes, the scatter ranges and probabilistic predictions for the desired response are also credible since the desired responses are obtained by repeated application of those deterministic methods/codes.

Returning to Fig. 3, note that uncertainties are introduced and predicted every where there is a probabilistic distribution schematic. On the syntheses part (left side), uncertainties enter from the constituent materials and any successive upward scale up to the structural scale. On the decomposition side (right side), uncertainties are traced at any successive downward scale down to the constituent material, where failure modes are easily identified and respeclive fracture criteria are readily implemented. Another important point to note is that the 


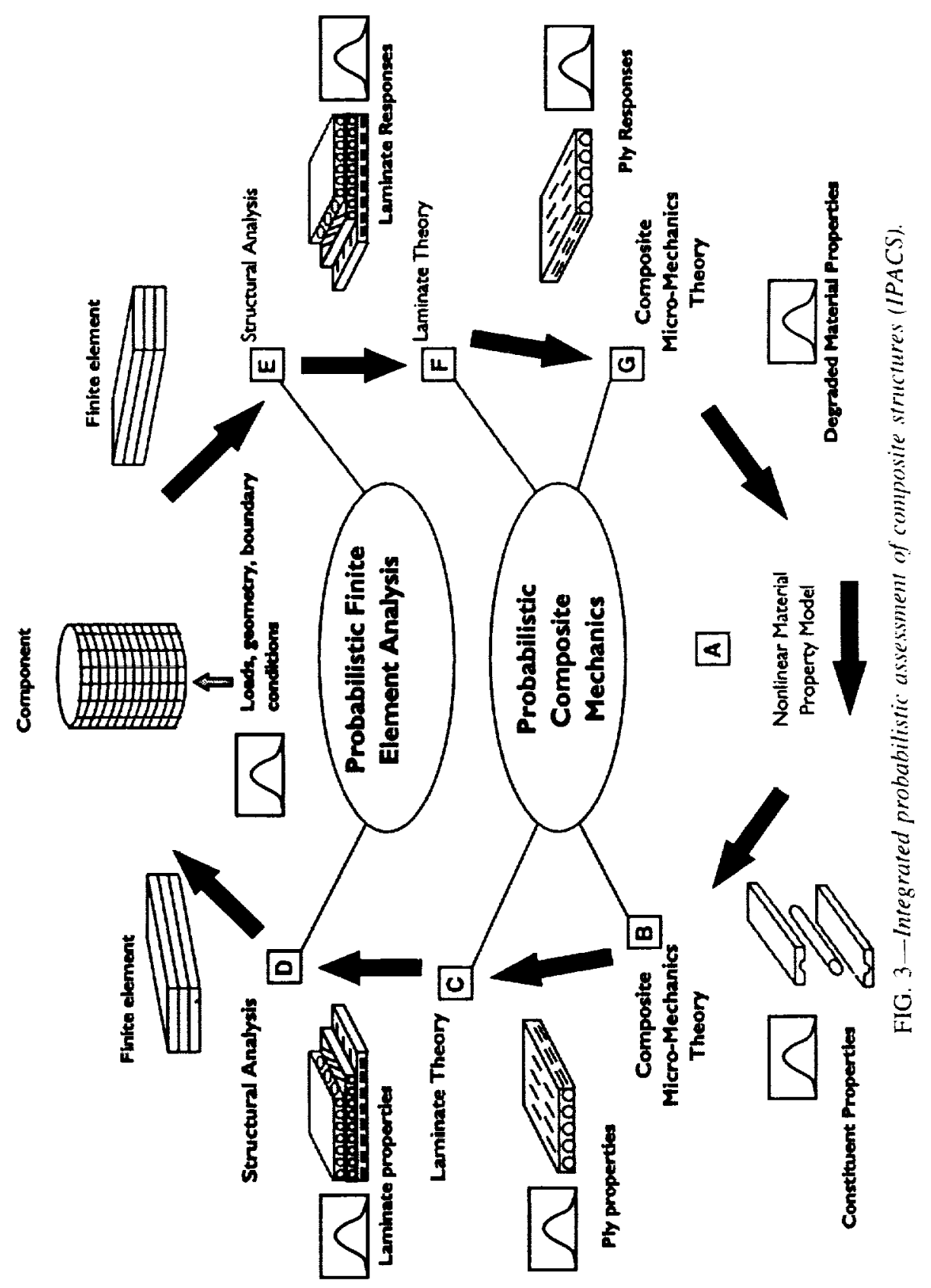


structural model can be relatively coarse but be inclusive of the whole structure, that is, the structural model must be inclusive of the boundary, loading, and environmental conditions.

\section{Verification}

Verification results will be presented for unidirectional tensile and compressive strengths, strength of specimens with a hole, and panel shear buckling. The verification results were obtained by different researchers as noted in the acknowledgment.

The ground rules werc: (1) predict the probabilistic distribution curve by including their knowledge of the scatter range and the mean values in all the primitive variables, and (2) plot their experimental data on the respective predicted probability distribution curve. The composite system used in the verifications is graphite fiber/epoxy. Typical properties for the graphite fibers, the scatter, and distributions used in the simulations are listed in Table 1 . It is noted that some of the strengths were modified to match proprietary data. Those for the matrix are listed in Table 2. All the comparisons discussed subsequently are for simulation results obtained by using the information in Tables 1 and 2 and from composites made from the same or very similar constituent materials.

\section{Ply Tensile Strength}

One of the important properties in composite design is tensile strength. It is important to know how well probabilistic predictions compared with measured data. The comparisons are

TABLE 1-Uncertainties in the fiber constituent properties.

\begin{tabular}{|c|c|c|c|}
\hline Fiber & Mcan & Assumed Scalter, $\%$ & Distributicn Type \\
\hline Normal modulus, $E_{j 1 i}$ & $213.7 \mathrm{GPa}$ & 5 & Normal \\
\hline Normal modulus, $E_{122}$ & $13.8 \mathrm{GPa}$ & 5 & Normal \\
\hline Poisson ratio, $v_{112}$ & 0.20 & 5 & Normal \\
\hline Poisson 's ratio, vt?2 & 0.25 & 5 & Normal \\
\hline Shear modulus, $G_{112}$ & $13.8 \mathrm{GPa}$ & 5 & Normal \\
\hline Shear modulus, $G_{12}$ & $6.9 \mathrm{GPa}$ & 5 & Normal \\
\hline Tensile strength, $S_{j t}$ & $3.3 \mathrm{GPa}$ & 5 & Weibull \\
\hline Conpressive strength, $S_{\text {, }}$ & $3.0 \mathrm{GPa}$ & 5 & Weibull \\
\hline
\end{tabular}

Notr: $1.0 \mathrm{GPa}=0.145138 \mathrm{Mpsi}$.

TABIE 2-Uncertainties in the matrix constituent properties.

\begin{tabular}{|c|c|c|c|}
\hline Fiber & Mean & Assumed Scatter, $\%$ & Distribution Type \\
\hline Normal modulus, $E_{m}$ & $3.4 \mathrm{GPa}$ & 5 & Normal \\
\hline Poisson's ratio, $\nu_{m}$ & 0.35 & 5 & Normal \\
\hline Tensile strength, $S_{m T}$ & $0.1 \mathrm{GPa}$ & 5 & Weibull \\
\hline Compressive strength, $S_{m c}$ & $0.24 \mathrm{GPa}$ & 5 & Weibull \\
\hline Shear strength, $S_{\text {独 }}$ & $0.09 \mathrm{GPa}$ & 5 & Weibull \\
\hline \multicolumn{4}{|l|}{ Fabrication variables: } \\
\hline Fiber volume ratio, $k_{I}$ & $60 \%$ & 2 & Normal \\
\hline Void volume ratio, $k_{f}$ & $2 \%$ & 5 & Normal \\
\hline Ply thickness, $t$ & $0.127 \mathrm{~mm}$ & 5 & Normal \\
\hline Ply misalignment & $0^{\circ}$ & $2^{a}$ & Normal \\
\hline
\end{tabular}



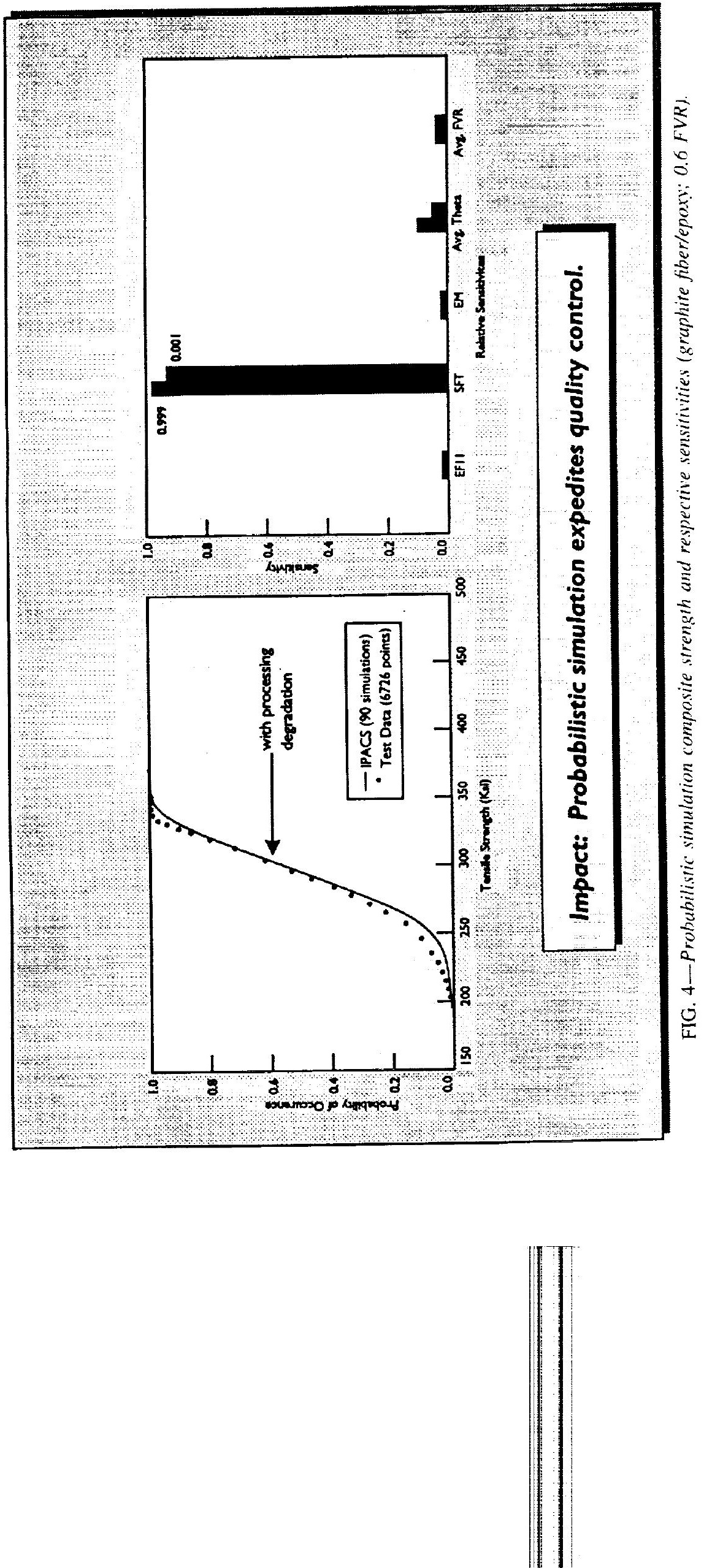
shown (Fig. 4) in terms of cumulative distribution function. Note the number of test data. Recall that the predicted curve was plotted first using information from Tables 1 and 2 and then the experimental dati. Obviously, the comparisons are excellent throughout the scatter range. Also in Fig. 4 are plotted the sensitivity factors for two probability levels 0.001 ( 1 in 1000) and 0.999 (999 in 1000).

Several observations are in order from the information in Fig. 4. (1) The scatter in the ply strength is considerable, spreading $1378 \mathrm{GPa}(200 \mathrm{ksi})$ to $2412 \mathrm{GPa}(350 \mathrm{ksi})$ with a mean ( 0.5 probahility) of about $2067 \mathrm{GPa}(300 \mathrm{ksi})$. This means that the probabilistic simulation captures: (a) the physics through the composite mechanics, (b) the scatter through the procedure described previously, and (c) the known and assumed information in Tables I and 2. (2) Any test value from the material within the scatter range is a legitimate data point and expected from the probabilistic simulation used. (3) We can limit the allowable for ply strength for robust designs to the $1378 \mathrm{GPa}(200 \mathrm{ksi})$ lower limit in the scatter with a reliability of one exceedence in 10000 . The lower limit allowable will definitely provide us with a robust but heavy and expensive design. (4) The sensitivity factors indicate that the tiber strength dominates, as is to be expected. Other factors, including fiber misalignment (avg. Theta) and fiber volume ratio (FVR), are negligible. This information translates simply-we need only to control the fiber tensile strength and relax the quality control requirements on the others which are essential in fabricating reliable, cheap, and fast products. (5) The order and magnitude of the sensitivity factors remain practically constant throughout the scatter range. This further reinforces that fiber tensile strength dominates at all probability levels. The important conclusion is that probabilistically predicted ply strengths following the procedure described hercin are credible and can reliably be used in composite design.

\section{Ply Compressive Strength}

Ply compressive strength is also imporlant in composite design. Comparisons are shown in Fig. 5. The predicted curve was plotted first and then the test data. Inputs for the predictions were from Tables 1 and 2 . The comparisons are excellent, demonstrating that the probabilistic simulation captures both the physics and the scatter. The scatter range is from $1240 \mathrm{GPa}(180 \mathrm{ksi})$ to $2205 \mathrm{GPa}(320 \mathrm{ksi})$ with a mean of about $1722 \mathrm{GPa}(250 \mathrm{ksi})$. Several factors contribute significantly to ply compressive strength (sensitivity, factors plot). The order of significance is liber volume ratio (FVR), matrix compressive strength (SMC), matrix shear modulus (GM), and fiber misalignment (Theta).

The multitude of these factors explain in part why it is difficult to interpret ply compressive strength test dita as well as having a consensus on a deterministic model. The respectable magnitude of Theta (fiber misalignment) lends credence to the deterministic micromechanics models based on kink bends of fiber. The other observations (except sensitivity factors) made for the ply tensile strength apply to compressive strength as well. The very important conclusion is that probabilistically predicted ply compressive strengths via the procedure described herein are credible and can reliably be used in design. Two points make these predictions very important. (1) The environmental (temperature and moisture) effects can also be reliably predicted since these effects influence the matrix only and since they are accounted for in the deterministic composite mechanics which are constituent level micromechanics based. Stated differently, temperature and moisture affect the matrix shear modulus and matrix compressive strength, both of which are significant sensitivity factors. (2) We do not need to have the large number of experiments shown in Figs. 4 and 5 for verification. The author contends that we can get by with at most three so long as they are within the probabilistically predicted scatter. 


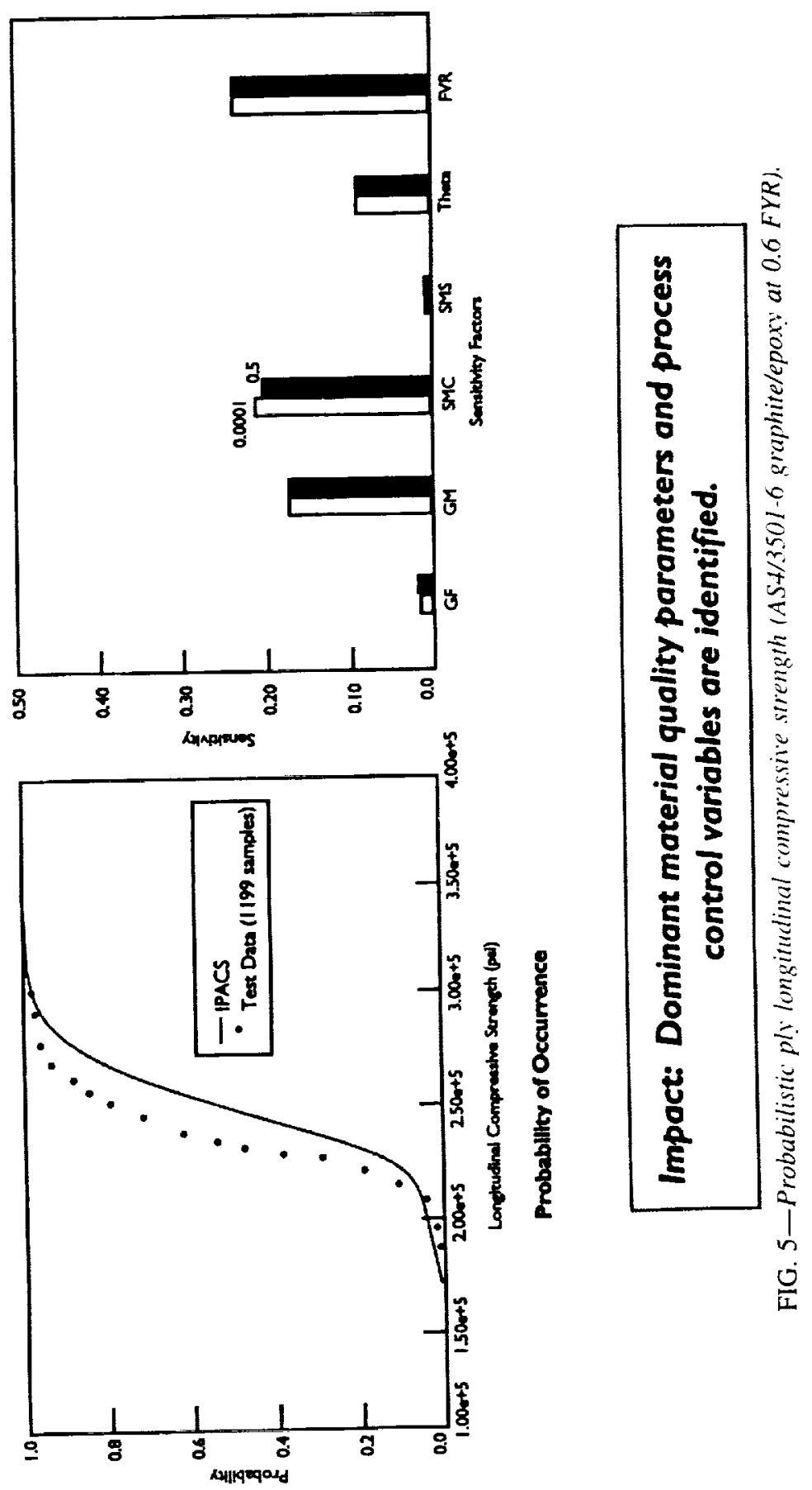


Tensile Strength of Laminate with a Hole

Laminate-with-a-hole tensile strength is a commonly used design allowable in tensile load bearing composite components. The tensile strength of a specific laminate with a hole predicted probabilistically is shown in Fig. 6 in terms of cumulative probability distribution. Three data points (see Acknomledgments) are also plotted. The data fall on the predicted curve. This is very important because it collectively verifies the probabilistic simulation for (1) composite laminate behavior, (2) stress concentration via finite element, (3) laminate fracture, and (4) the author's previous contention that three tests are sufficient for verification.

The important conclusions are (1) probabilistically predicted laminate strengths with stress concentrations are credible and can reliably be used for design of load-bearing composite structures with defects, and (2) only limited data ( 3 points minimum) are enough to verify laminate behavior and even composite component structural behavior provided that all three points fall on the predicted distribution. It is worth noting that the contention for three tests, mentioned in the previous section, was made long before the data in Fig. 6 became available.

Panel Shear Buckling

It is well known that composite panel shear huckling is important in aircraft fuselage and wing skin designs. The importance of composite panel shear buckling increases in importance in view of the difficulties, costs, and time associated with performing the requisite

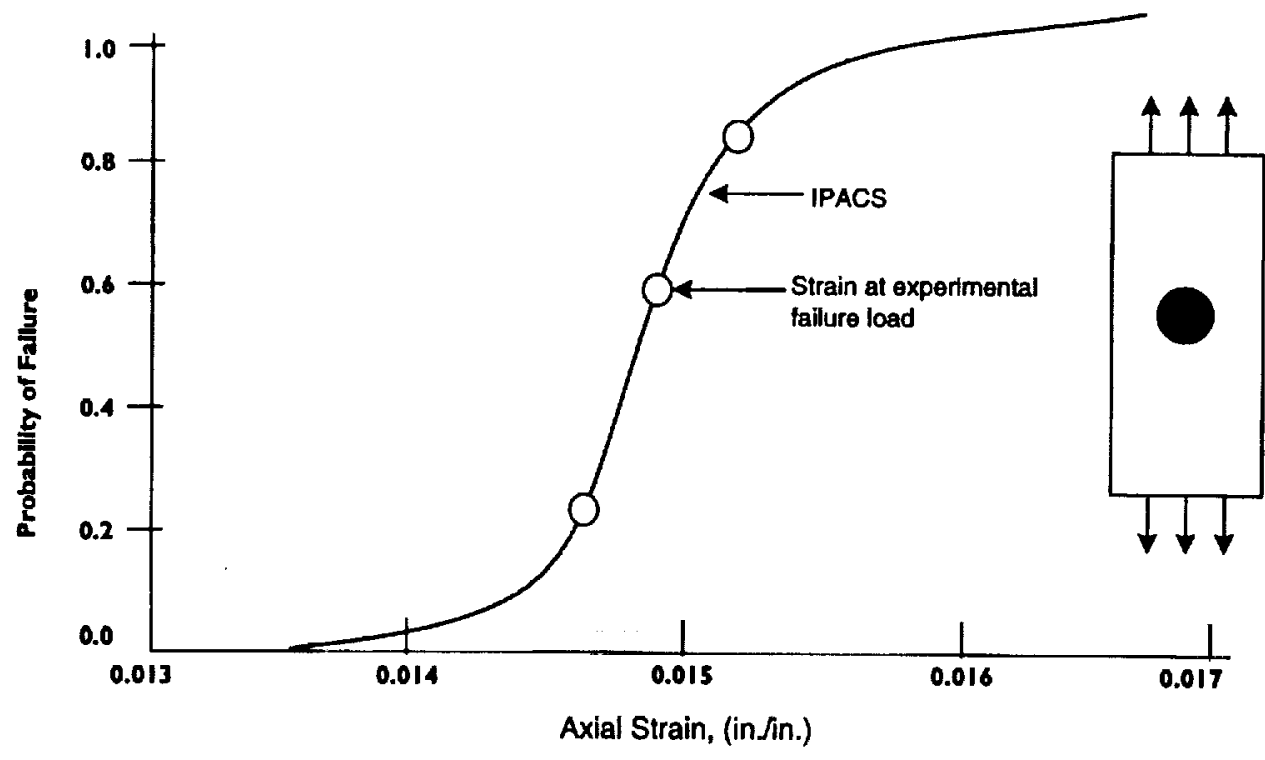

Impact: Design allowables can be set via probabilistic simulation with limited data.

FIG. 6-Probabilistic simulation accuracy predicts the near-field fracture strain scatter in composite laminates with holes (AS/EP [O/ $\pm 45 / 90]_{2 . s}$ composite). 
tests for design concepts verification. Therefore, verifiable probabilistic predictions will go a long way to minimize the testing that may otherwise be required.

Probabilistically predicted results for shear buckling of a stringer-reinforced composite panel are shown (Fig. 7) in terms of mean values and one and two standard deviations on either side of the mean. The predictions were based on the constituent material properties and their respective scatter ranges (Tables $I$ and 2 ). The probabilistic simulation for the panel shear buckling was performed by using the IPACS computer code. Experimentally available data (see Acknowledgments) were plotted in terms of vertical bars in the same figure. All the test data are within the predicted two-standard deviations scalter.

Ply thickness is by far the most sensitive factor to the panel's shear buckling, stringer locations (bay width left and right) and ply orientation having relatively minor significance compared to ply thickness. The implication is to tighten tolerances on ply thickness and relax the tolerances (1) for stringer precise location and (2) on ply orientation during the fabrication of those types of structures. The important conclusions are: (1) probabilistically predicted complex structural responses are credible and can be reliably used when obtained by procedures described herein; (2) factors that influence these responses are identifiable and can be appropriately adjusted for cost and time benefits without sacrificing reliability, and (3) probabilistically evaluated composite design concepts require relatively minimum testing. (The author will be presumptuous and call for only one test.)

\section{Select Extensions}

Assuming that verification demonstration results presented and discussed previously establish confidence in probabilistic simulation of composites, we proceed to describe three important extensions. These extensions are included as being representative of what can be done by using probabilistic simulation. Specifically, we will describe composite fabrication implications, testing guidelines, and laminate fatigue.

\section{Fabrication Implications}

In composite structural design, a commonly asked question is in situ strength versus pristine strength, especially for fiber tensile strength. Herein we illustrate how probabilistic simulation can be used to identify whether the fabrication process degrades fiber tensile strength and how much. We illustrate the procedure with the aid of Fig. 8, where two probabilistically predicted curves are plotted for ply tensile strength and the test data. Note that the two predicted curves are labeled as pristine and in situ. The pristine curve was obtained by using the fiber suppliers fiber mean tensile strength of $3721 \mathrm{GPa}(540 \mathrm{ksi})$ and the assumed scatter shown in Table 1 . The in situ fiber strength was obtained by degrading the fiber tensile strength by the difference in the means between the supplier of $3721 \mathrm{GPa}$ $(540 \mathrm{ksi})$ and the data of $3307 \mathrm{GPa}(480 \mathrm{ksi})$. Even though the supplier may claim no processing degradation, the data show otherwise.

The degradation of $413 \mathrm{GPa}(60 \mathrm{ksi})$ is believable because of the following. We note that the shape of the cumulative distribution functions is exactly the same for both the data and the predicted curves using the pristine fiber tensile strength and the assumed scatter. This implies that the scatter is properly captured and the two can be made to coincide simply by a parallel shift. It is important to note that the distributions of the pristine properties of the primitive variables are not needed. The reason is that different distributions can be assumed and re-perform the simulations. In this case the distributions assumed were sufficient because of the good agreement with the data in the ply tensile strength. The amount of parallel shift required usually equals the mean in one of the dominant primitive variables, in

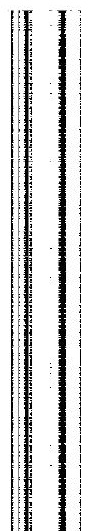



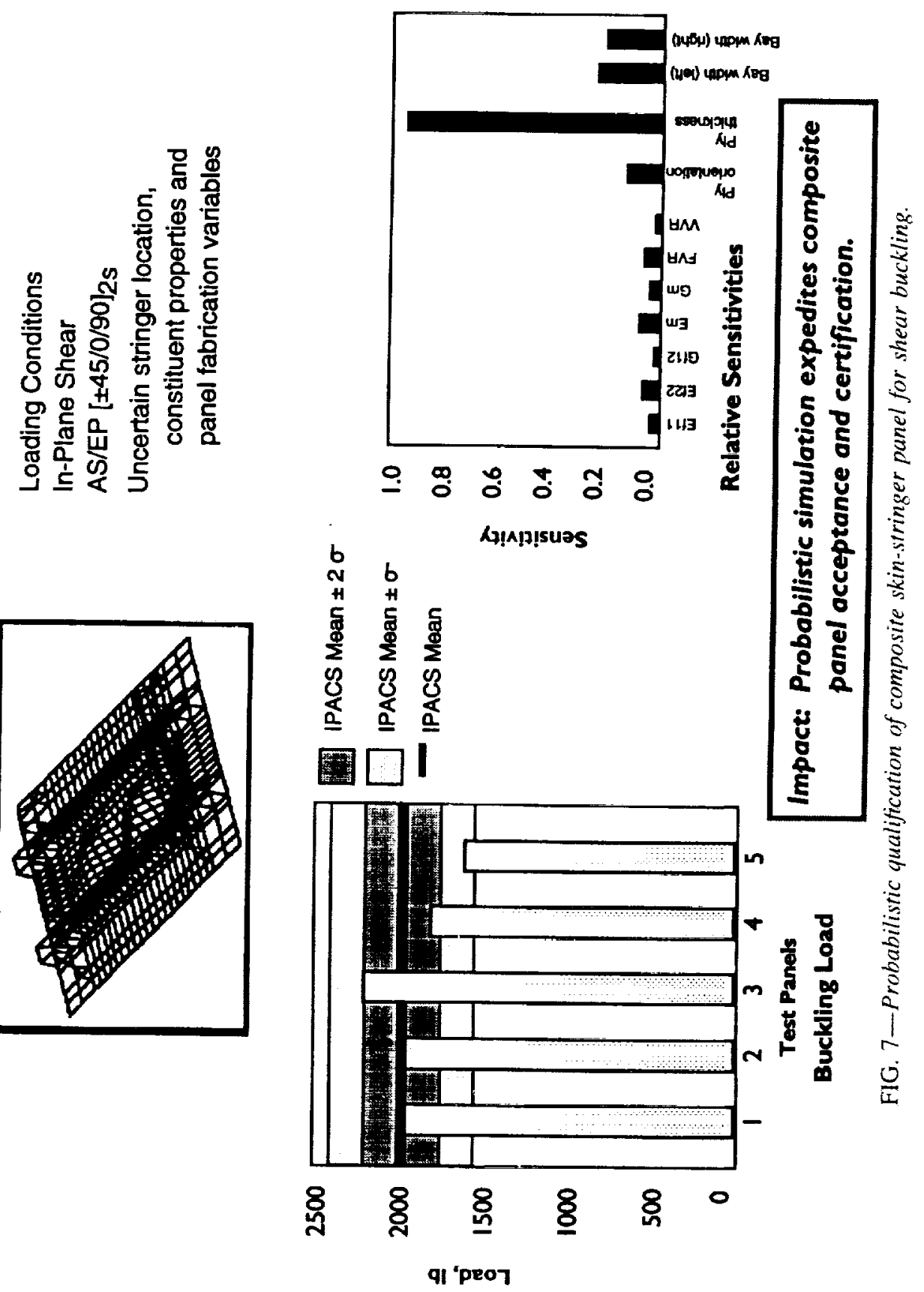


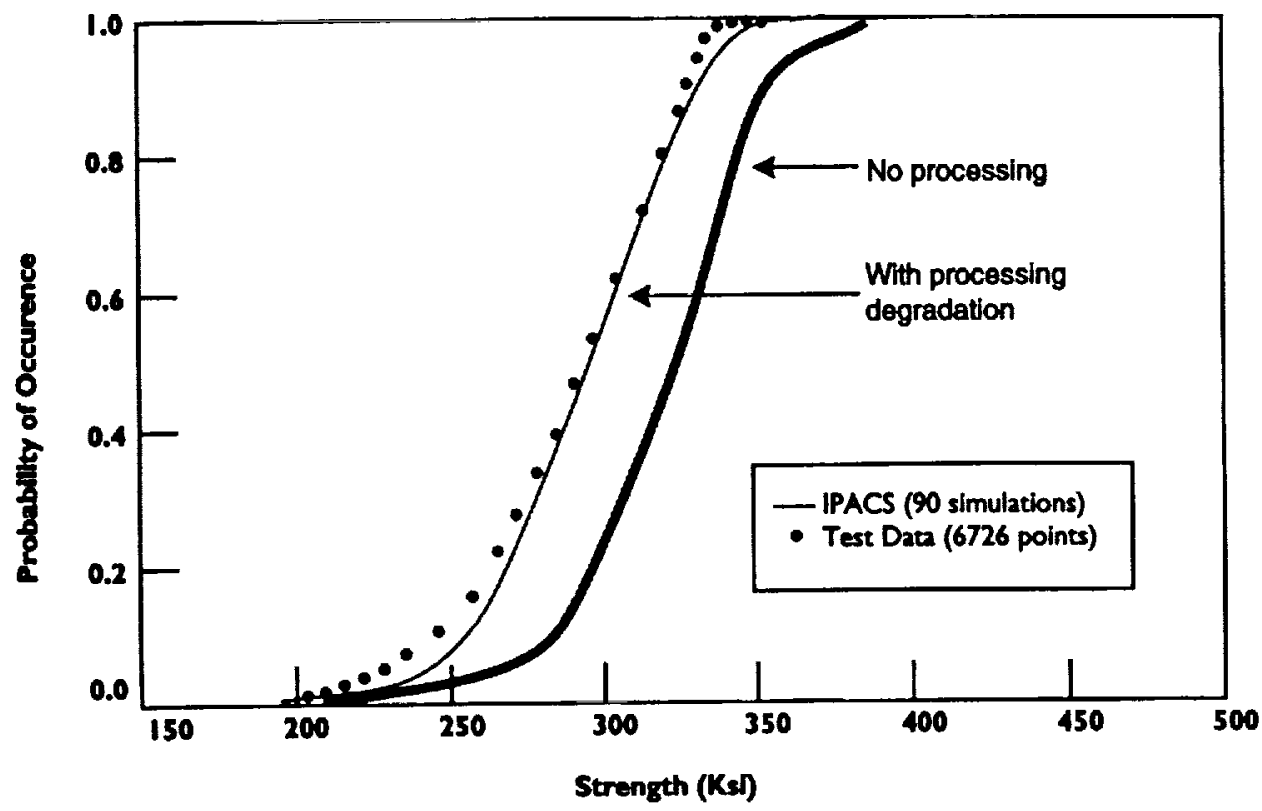

Impact: Probabilistic simulation quantifies the
degradation effects due to processing.

FIG. 8-Probabilistic simulation of longitudinal tensile strength scatter in AS/E composites.

this case, fiber tensile strength. Shifting is very important in performing and or verifying probabilistic simulations. For example, (I) missing a significant primitive variable will result in a similar shift, and (2) probabilistic structural responses obtained from coarse mesh finite element models can be calibrated by using the respective mean results from fine mesh finite element models [8]. The important conclusion from the above discussion is that probabilistic simulation can be effectively used to pinpoint fabrication processing effects on dominant primitive variables that are identifiable from respective sensitivity factors.

\section{Testing Guidelines}

Minimum reliable testing is critical in setting material allowances for composite structural design. Probabilistic simulation is adaptable to identify contributing factors and respective testing ramifications. What can be done and how is illustrated by the following specific example.

A single ply of about $12.7 \mathrm{~mm}$ ( 5 mils) thick and 0.6 fiber volume ratio will contain 15 fibers through its thickness. We probabilistically simulate the effect of the local fiber volume ratio on the ply tensile strength. The results obtained are shown in Fig. 9. As can be seen, the fiber volume ratio of fibers located near the quarter points of the thickness has a negligible effect as compared to that at the outer surfaces and at the center.

The implications from these results are: (1) Tensile strength specimens should either be pin loaded, fiber volume ratio graded through-the-thickness, or flat-wise long-tapered in order to transfer load directly and more uniformly to the inner fibers. The flat-wise long

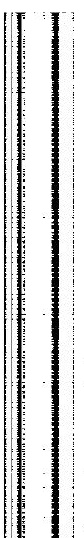




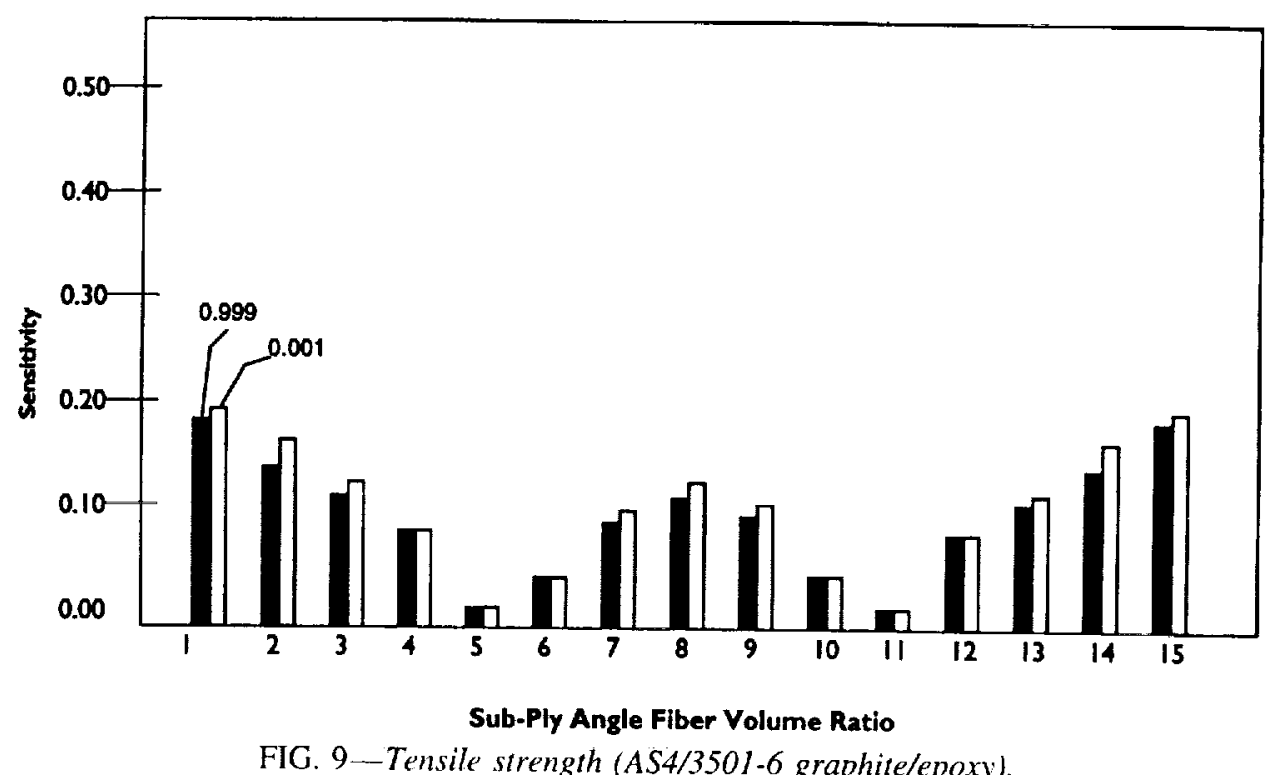

taper is the most practical. It is interesting to note that type of specimen was used by the author and one of his colleagues about 20 years ago to obtain high strength with reduced scatter in tensile strength tests of boron/aluminum specimens (unpublished data). Neither the author nor his long-since-retired colleague had the vaguest notion why it worked. The important conclusion is that probabilistic composite simulation can aid in identifying effective testing techniques and recommended guidelines.

\section{Laminate Fatigue}

Laminate fatigue is indispensable for any practical composite structural applications subjected to cyclic loading. Having demonstrated that probabilistic simulation credibly and reliably predicts composite ply laminate and structural behavior, it is interesting to explore its extension to predict composite laminate fatigue. Herein we brieffy describe how it can be done and show typical results obtained for a specific laminate. The details are described in Ref 9.

In order to probabilistically simulate laminate fensile fatigue, we postulate that (1) laminate fatigue strength is limited by first ply failure; (2) the respective ply strengths are degraded cyclically as determined by respective degradation in the constituent materials, that is, the strength of the constituent in that micro-stress component (note that any constituent synergism to fatigue resistance will be at a higher scale than the micromechanics scale); (3) the degraded properties are used at preselected laminate stresses while the laminate is loaded in tension and the number of cycles is increased until the first ply fails; (4) the number of cycles at first ply failure is taken as the laminate fatigue; and (5) the results are plotted as cumulative distribution functions. Results from this procedure are shown in Fig. 10 for a specific laminate cycled at $90 \%$ of its first ply static strength.

The fatigue (number of cycles) scatter is from 20 to about $50 \%$ of infinite life, which is assumed to be the mean number of cycles at $10 \%$ of first ply failure static strength. Note that the mean value is about $40 \%$ of the infinite life. The dominant sensitivities are in order of 

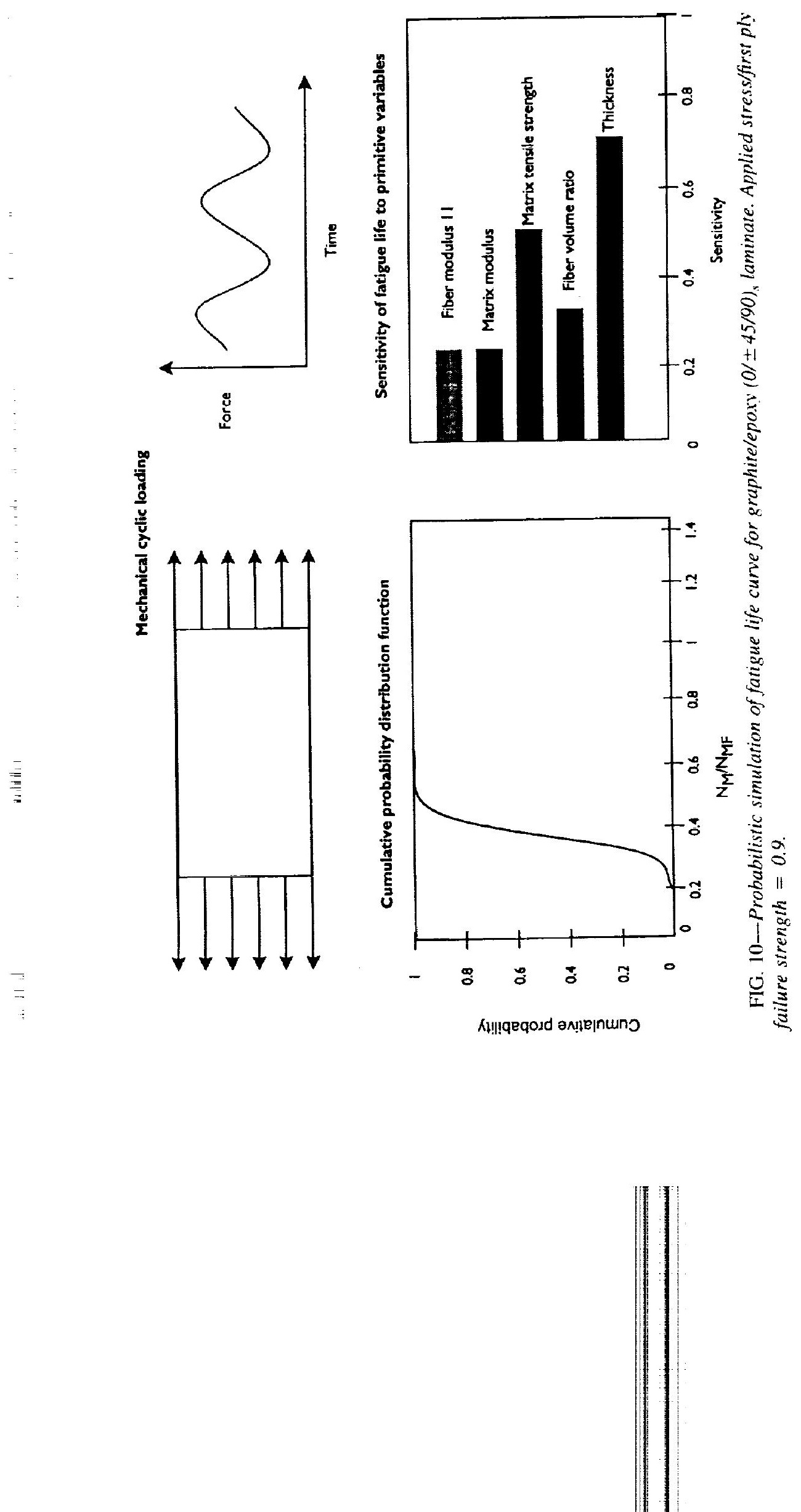
significance: ply thickness, matrix tensile strength, fiber volume ratio, fiber and matrix modulus. Implications are: (1) laminate fatigue exhibits substantial scatter, and (2) several primitive variables affect fatigue, making it difficult to interpret test data and set design allowables. The important conclusion is that laminate fatigue can be simulated probabilistically by procedures described herein. Respective verification comparisons are still pending, but the author conjectures they will be as successful as the comparisons for the static cases.

\section{Probabilistic Composite Design}

Probabilistic design is simply the evaluation of a composite structure for its probability of failure or its reliability. We described all the essentials required to perform a probabilistic design. In this section we illustrate the procedure with a specific example-the design of a composite radome.

The geometry and the finite element model are shown in Fig. 11. Note that the entire radome is modeled with a relatively coarse mesh. The laminate configuration selected is $[0 / \pm 45 / 90]_{\text {s }}$ (relative to meridian). Some inputs to the probabilistic design are shown in Table 3 with their respective scatter. The remaining constituent properties are from Tables 1 and 2. Using IPACS, the reliability of the radome was predicted to be 0.999999 or one failure in one million. The sensitivity factors for stress and strength are shown in Fig. 12. For low probability of failure (high reliability), the dominant primitive variables are fiber volume ratio and fiber modulus. Interestingly, the ply thickness and the applied pressure are

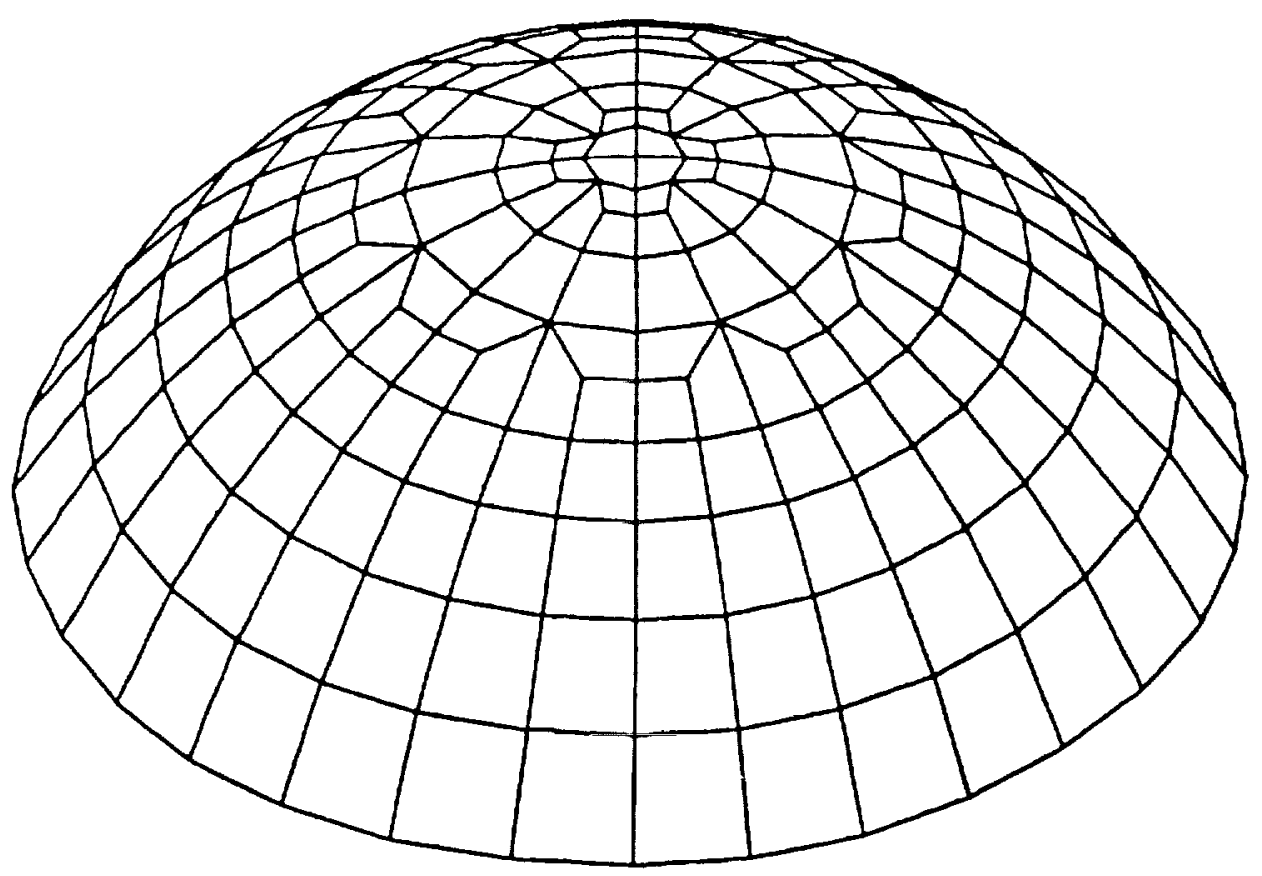

Finite Element Model

FIG. 11-Radome structure finite element model. 
TABLE 3-Random variables used in the example.

\begin{tabular}{lccc}
\hline Random Variable & Mean Value & Coefficient of Variation & Distribution Type \\
\hline Fiber modulus, $E_{f \prime 1}$ & $213.7 \mathrm{GPa}$ & $10 \%$ & Weibull \\
Fiber volume ratio & 0.6 & $10 \%$ & Lognormal \\
Thickness & $12.7 \mathrm{~mm}$ & $5 \%$ & Normal \\
Air pressure & $55.2-96.5 \mathrm{~Pa}$ & $25 \%$ & Normal \\
\hline
\end{tabular}

Note: $1.0 \mathrm{GPa}=0.145138$.

$1.0 \mathrm{~mm}=0.394$ mils.

$1.0 \mathrm{~Pa}=1.45 \times 10^{+1} \mathrm{psi}$.

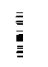

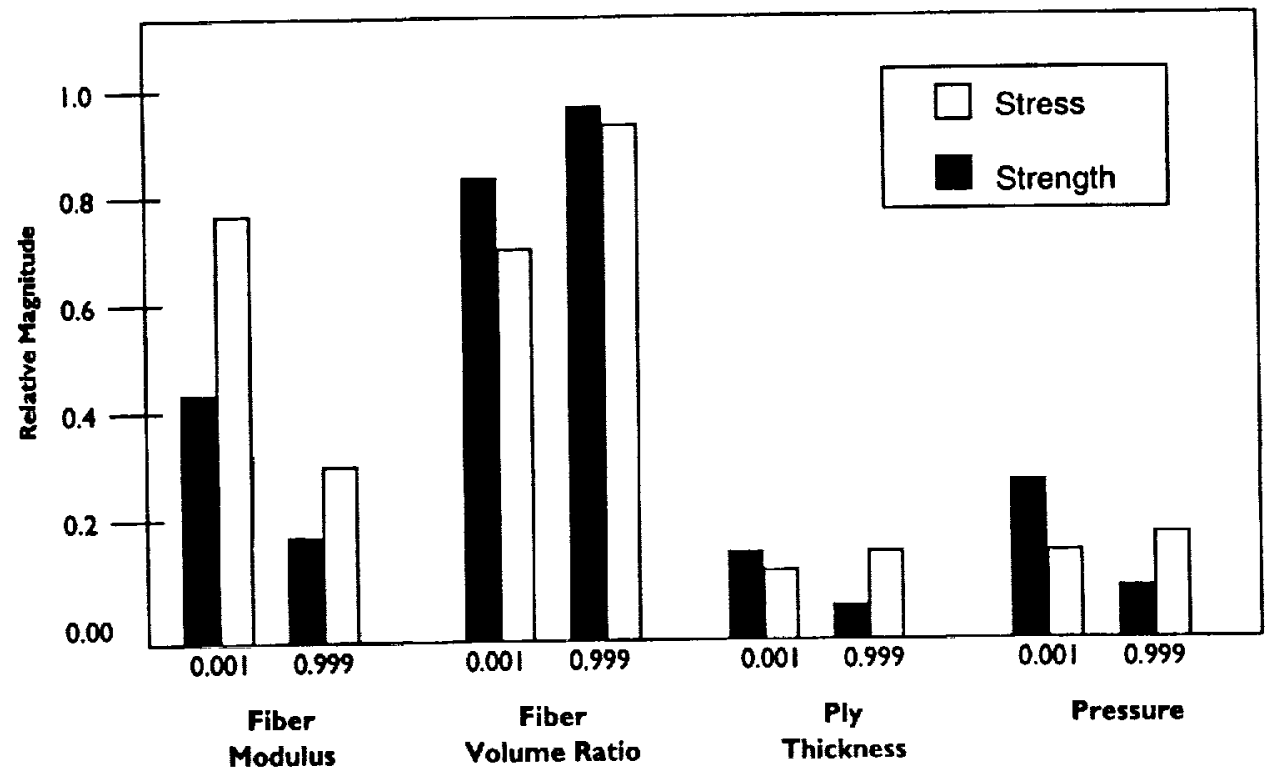

FIG. 12-Probabilistic sensitivity factors affecting stress and strength of the redome.

relatively insignificant at high probability of failure (low reliability) while the fiber volume ratio dominates (see also Ref 10 ).

Implications: (1) design for high reliability by controlling the dominate primitive variables at low levels of failure probability, (2) design for proof testing by controlling the primitive variables with dominant sensitivities at high levels of failure probability, and (3) each design needs to be evaluated individually for reliability and dominant sensitivities since results may not be transferrable. The important conclusion is that composite structures can be probabilistically designed by the procedures described herein.

\section{Summary}

Probabilistic methods and computer codes have been described for composite structural design starting from constituent materials and tracking the design evolution to structural configuration. Verification comparisons were presented to lend credence to probabilistic design. The salient results are summarized as follows: 
1. Probabilistic simulation has been verified for ply, tensile, and compressive strength; for laminate with a hole tensile strength; and for shear buckling of a skin stringer reinforced panel. The experimental data are within the predicted distribution for all cases.

2. Dominant probabilistic primitive variables and sensitivity factors have been identified for all verification cases.

3. Probabilistic simulation is effective in identifying in situ strength and testing guidelines to minimize scatter-fiber tensile strength degrades by $10 \%$ and a flat-wise tapered specimen is most suitable for ply tensile strength.

4. Probabilistically evaluated laminate fatigue life has substantial scatter-20 to $50 \%$ of respective infinite life when cycled at $90 \%$ of its static strength.

5. Probabilistic structural evaluation of a composite radome was designed with a reliability of 0.999999 or accepting one failure in one million.

6. Primitive variables with dominant sensitivities at low probability of failure must be controlled to assure high reliability designs, while those with dominant sensitivities at high probabilities of failure must be controlled for effective proof testing

\section{Acknowledgme'nts}

The author expresses his gratitude to Dr. Larry Sobel from Northrop-Grumman and to Dr. Steve Wanthel from McAir for their many helpful suggestions during the development of the IPACS computer code and for performing the verification comparisons presented in the text. He also expresses his gratitude to his NYMA colleagues for developing the IPACS computer code and for generating the computational simulation results included in the article.

It is noted that composite structural analysis and/or design has been a continuing activity of the author with various colleagues throughout his professional career. The extension to formally incorporate uncertainties in the design, in order to evaluate respective reliabilities, has been an aggressively pursued activity over the past 12 years. The formal methods, computer codes, interpretations, implications, and conclusions are necessarily continuing accumulations of that collective experience which cannot conveniently be referenced without recourse to a full bibliography.

\section{References}

[I] Shiao, M. C., Abumeri, G.H., and Chamis, C. C., "Probabilistic Assessment of Composite Structures," AIAA/ASME/ASCE/AHS/ASC, Proceeding.s, 34th Structures, Structural Dynamics and Materials Conference, 1993, AIAA, New York, pp. 1174-1186.

[2] Shiao, M.C. and Chamis, C. C., "Probabilistic Design of Smart Composite Structures," Proceedings, 39th International SAMPE Symposium, Califomia, 1994, Vol. 39, SAMPE, Covina, CA. pp. $3112-3126$.

[3] Thacker, B. and Wu, Y.-T., "A New Response Surface Approach for Structural Reliability Analysis," AIAA/ASME/ASCE/AHS/ASC, Proceedingr. 33rd Structures, Structural Dynamics and Materials Conference, Dallas, 1992, AIAA, New York, pp. 586-593.

[4] Murthy, P. L. N. and Chamis, C. C., "ICAN: Integrated Composites Analyzer," Journal of Composites Technology \& Research, Vol. 8, No. 1, pp. 8-17.

[5] Stock, T. A., Belline, P. X., Murthy, P. L. N., and Chamis, C. C., "Probabilistic Composite Micromechanics," AIAA/ASME/ASCE/AHS, Proceedings, 29th Structures, Structural Dynamics and Materials Conference, Part 3, 1988, AIAA, New York, pp. 1289-1298.

[6] Mase. G. T., Murthy, P. L. N., and Minnetyan, L., "Quantification of Scatter in Composite Properties Via Probabilistic Mechanics," Composite Material Technology, PD-Vol. 45, ASME, New York, 1992, pp. $71-76$.

[7] Wu, T.-T., "NESSUS Structural Reliability Analysis Methods-Review and New 
Developments," AIAA/ASME/ASCE/AHS/ASC, Proceedings, 33rd Structures, Structural Dynamics and Materials Conference, Dallas, 1992, AIAA, New York.

[8] Shiao, M. C. and Chamis, C. C., "Mapping Methods for Computationally Efficient and Accurate Structural Reliability," AIAA/ASME/ASCE/AHS/ASC, Proceedings, 33rd Structures, Structural Dynamics and Materials Conference, Dallas, 1992, AIAA, New York, pp. 433-443.

[9] Shah, A. R., Murthy, P. L. N., and Chamis, C. C., "Effect of Cyclic Thermo-Mechanical Load on Fatigue Reliability in Polymer Matrix Composites," Proceedings, 36th AIAA/ASEE Conference, New Orleans, LA, April 1995, Part 3, AIAA, New York, pp. I670-1682.

[IO] Shah, A. R. and Liaw, D. G., "Probabilistic Sizing of Laminates with Uncertainties," Proceedings, 24th International SAMPE Technical Conference, Toronto, Canada, 1992, Vol, 24, SAMPE, Covina, CA, pp. T447-T460. 